\title{
Idosos mais idosos: uma revisão da literatura
}

\author{
Older senior: a review of literature
}

Ancianos Ancianos: una revisión de la literatura

Priscila de Paula Marques

Carolina Neves Freiria

Caroline Nunes Santiago

Matilde Côrrea

Sandra Aparecida Bratifische

Priscila Maria Stolses Bergamo Francisco

Maria José D’Elboux

RESUMO: O objetivo é revisar os artigos científicos publicados nos últimos cinco anos sobre idosos longevos ( $\geq 80$ anos) e identificar o perfil dessas publicações. Foi realizada uma revisão da literatura, utilizando-se a Biblioteca Virtual em Saúde. A busca resultou na seleção de 36 artigos nacionais e internacionais. Observou-se que a maioria dos estudos era do tipo transversal, realizados em países da Europa e com maior participação de idosos do sexo feminino. Câncer e doenças cardíacas foram os temas mais abordados.

Palavras-chave: Envelhecimento; Idoso de 80 anos ou mais; Saúde do idoso. 
ABSTRACT: The objective is to review the scientific articles published in the last five years on long-lived elderly ( $\geq 80$ years) and to identify the profile of these publications. An review of literature was carried out, using the Virtual Health Library. The search results in the selection of 36 national and international articles. It was observed that most of the studies were of a cross-sectional type, in countries of Europe and a greater participation of the elderly women. Cancer and heart disease were the topics most covered.

Keywords: Aging; Aged, 80 and over; Health of the Elderly.

RESUMEN: El objetivo es revisar los artículos científicos publicados en los últimos cinco años sobre personas mayores de larga vida ( $\geq 80$ años) e identificar el perfil de estas publicaciones. Se realizó una revisión de literatura utilizando la Biblioteca Virtual en Salud. La búsqueda resultó en la selección de 36 artículos nacionales e internacionales. La mayoría de los estudios fueron estudios transversales realizados en países europeos con una mayor participación de mujeres mayores. El cáncer y las enfermedades cardíacas fueron los temas más discutidos.

Palabras clave: Envejecimiento; De 80 años en adelante; Salud de los ancianos.

\section{Introdução}

O envelhecimento populacional é um fenômeno mundial que ocorre em consequência, principalmente, da queda da taxa de mortalidade e redução da taxa de natalidade (IBGE, 2015). A proporção de pessoas mais velhas evidencia que este é o segmento populacional que cresce mais rápido no mundo, sendo que, no caso dos idosos mais idosos ( $\geq 80$ anos), este segmento possui ritmo mais acelerado quando comparado com aquele de idosos mais jovens (60 a 69 anos) (UN, 2013). Segundo a Organização Mundial da Saúde (2015), “O envelhecimento da população é um dos maiores triunfos da humanidade e também um dos nossos grandes desafios".

Em países em desenvolvimento, do ponto de vista cronológico, consideram-se idosos aqueles indivíduos com idade igual ou superior a 60 anos, sendo denominados “idosos jovens" os indivíduos que pertencem à faixa etária entre 60 e 69 anos; “idosos 
idosos", aqueles com idade entre 70 e 79 anos e "idosos velhos" ou com "velhice avançada" os idosos com idade igual ou superior a 80 anos (Lima, 2014).

Entende-se por envelhecimento longevo a continuidade do processo fisiológico após os 80 anos, em que há aceleração da perda da capacidade funcional e cognitiva. Os indivíduos nessa faixa etária possuem maior risco de envelhecer, o que é acompanhado por múltiplas doenças crônicas não transmissíveis (DCNT) e outros agravos à saúde. Aproximadamente $50 \%$ da parcela de indivíduos com idade $\geq 90$ anos são acometidos pela Doença de Alzheimer ou outras doenças neurodegenerativas, com progressiva perda de habilidades cognitivas e funcionais, trazendo elevados gastos em saúde (Baltes, \& Smith, 2006; Kumon, Silva, VP, Silva, AI, \& Gomes, et al., 2009).

Contudo, ressalta-se que, no grupo de idosos mais longevos, há grande heterogeneidade de características. Enquanto os octogenários manifestam doenças degenerativas e passam anos vivendo com alto grau de dependência, alguns centenários não apresentam grandes incapacidades e chegam à idade extrema sem morbidades importantes. Além dessas diferenças, do ponto de vista genético, o processo de envelhecimento dos órgãos dos centenários é mais lento (Kumon, Silva, VP, Silva, AI, \& Gomes, 2009). Apesar do aumento exponencial de idosos longevos na estrutura recente da população, evidenciam-se lacunas importantes na produção de conhecimento; com isso, estas considerações justificam o interesse em desenvolver levantamentos bibliográficos e revisões da literatura, sobre a produção científica relacionada ao envelhecimento, em especial com os idosos mais idosos, considerando-se que essa tendência demográfica acarreta implicações políticas, sociais e econômicas no acesso e atendimento à saúde. Portanto, o objetivo deste estudo é revisar os artigos científicos publicados nos últimos cinco anos sobre idosos longevos (>=80 anos) e identificar o perfil dessas publicações.

\section{Métodos}

O presente estudo utiliza o método da revisão da literatura que permite a busca, análise crítica e a síntese de evidências disponíveis sobre o tema, permitindo identificar lacunas que direcionam para realização de futuras pesquisas sobre o assunto baseadas na prática em evidências (Gil, 2002). 
Para a operacionalização desta revisão, foram utilizadas as seguintes etapas tendo em vista a identificação, seleção, elegibilidade e inclusão dos estudos na revisão: seleção da questão temática; definição das bases de dados e descritores; estabelecimento dos critérios de inclusão e exclusão das publicações para definir a amostra; determinação das informações retiradas dos artigos, análise e avaliação dos trabalhos selecionados na revisão e interpretação dos resultados (Souza, Silva, \& Carvalho, 2010).

O levantamento bibliográfico desta revisão foi realizado no mês de setembro de 2017 através da Biblioteca Virtual em Saúde (BVS) e a partir da seguinte questão norteadora: "Qual conhecimento produzido sobre os idosos mais idosos, entre os anos de 2013 e 2017?". Foi utilizado o descritor “idosos com 80 anos ou mais", consultandose as seguintes bases indexadoras: Medical Literature Analysis and Retrieval System Online (MEDLINE) e Literatura Latino-Americana e do Caribe em Ciências da Saúde (LILACS). Foram adotados critérios de inclusão e exclusão para a recuperação dos trabalhos. Os critérios de inclusão utilizados para a seleção das amostras foram: artigo científico com texto na íntegra; nos idiomas português, inglês ou espanhol; publicados nos últimos cinco anos (2013-2017). Excluíram-se trabalhos como teses e dissertações, a fim de eliminar publicações que não passaram por rigorosa avaliação e revisão por pares. Também foram excluídos artigos de revisão e duplicados, compondo ao final uma amostra de 36 artigos para a análise.

Após o levantamento das publicações, os resumos foram lidos e analisados, segundo os critérios de inclusão/exclusão estabelecidos. Após a leitura dos resumos, os trabalhos selecionados foram lidos e analisados na íntegra e em profundidade. Em seguida, procedeu-se ao fechamento dos mesmos, de acordo com formulário delineado para essa finalidade, de modo a dar visibilidade aos principais atributos de cada produção (autor, local e ano de publicação, periódico, áreas temáticas, objetivos, tipo de estudo, amostra e resultados).

\section{Resultados e Discussão}

Foram identificadas 140 publicações após a busca bibliográfica. Destas, foram excluídas 93, após analisar e obedecer rigorosamente aos critérios de inclusão e exclusão deste estudo. Foram selecionados para a análise 47 artigos e, após a leitura dos 
títulos e resumos, tornaram-se elegíveis 36 artigos (9-44), para leitura completa e análise.

A Figura 1 apresenta o fluxo de seleção dos estudos

Figura 1. Fluxo de seleção dos estudos

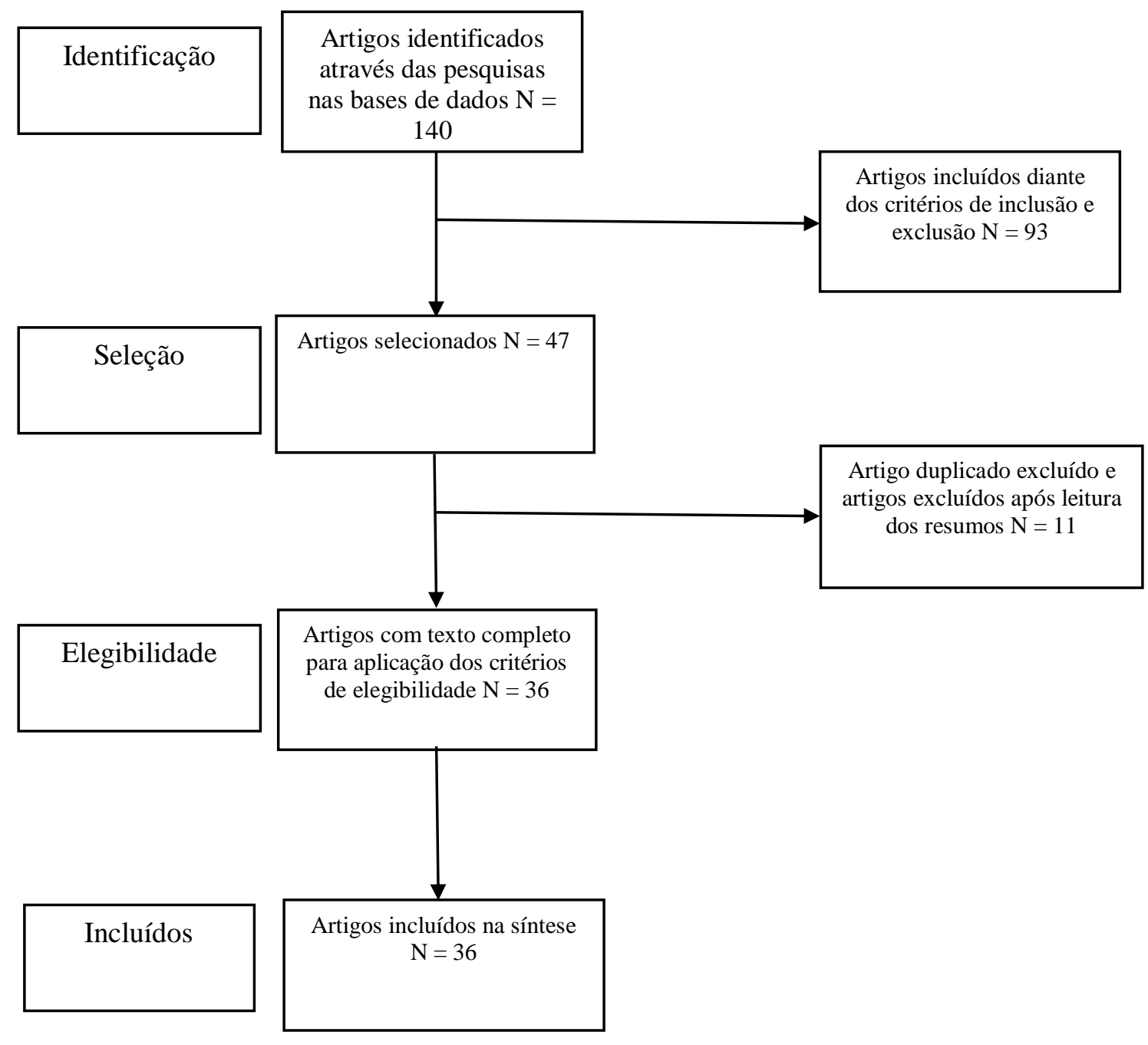




\section{Levantamento Bibliográfico}

Empregando-se os critérios de inclusão e exclusão adotados, foram localizados 36 trabalhos qualificados. A base de dados na qual foi encontrado maior número de publicações foi a MEDLINE. Esse resultado mostra a maior produção internacional de artigos científicos sobre idosos longevos. A descrição dos artigos publicados na literatura, de acordo com as variáveis pesquisadas no período de 2013 a 2017 é apresentada no Quadro 1.

Quadro 1. Descrição dos artigos publicados na literatura, de acordo com as variáveis pesquisadas no período de 2013 a 2017

\begin{tabular}{|c|c|c|}
\hline Autor/Ano/País & Periódico & Objetivo do estudo \\
\hline $\begin{array}{l}\text { Bode, B., et al. (2015). } \\
\text { Estudo multicêntrico em } \\
17 \text { países (9) }\end{array}$ & $\begin{array}{l}\text { Diabetes, Obesidy } \\
\text { and Metabolism }\end{array}$ & $\begin{array}{l}\text { Avaliar pacientes com idade entre } 55 \text { e } 80 \\
\text { anos com diabetes mellitus tipo } 2\end{array}$ \\
\hline $\begin{array}{l}\text { Bonicoli, et al. (2016). } \\
\text { Itália (10) }\end{array}$ & $\begin{array}{c}\text { Injury/ Elsevier } \\
\text { Journal }\end{array}$ & $\begin{array}{l}\text { Comparar a atividade da vida diária e } \\
\text { mortalidade em pacientes com } \geq 80 \text { anos } \mathrm{e} \\
\text { fratura de quadril }\end{array}$ \\
\hline $\begin{array}{l}\text { Bourguignon. L., et al. } \\
\text { (2016). França (11) }\end{array}$ & $\begin{array}{l}\text { Antimicrobial Agents } \\
\text { and Chemotherapy }\end{array}$ & $\begin{array}{l}\text { Propor um modelo capaz de prever a } \\
\text { farmacocinética da vancomicina em pessoas } \\
\text { muito idosas }\end{array}$ \\
\hline $\begin{array}{l}\text { Bruyère, O., et al. } \\
\text { (2014). Estudo } \\
\text { multicêntrico em } 9 \\
\text { países (12) }\end{array}$ & $\begin{array}{l}\text { Archives of } \\
\text { Gerontoloy } \\
\text { Geriatrics }\end{array}$ & $\begin{array}{l}\text { Avaliar a prevalência da inadequação da } \\
\text { vitamina D nas mulheres europeias com } \geq \\
80 \text { anos. }\end{array}$ \\
\hline $\begin{array}{l}\text { David, C. N., et al. } \\
\text { (2016). Brasil (13) }\end{array}$ & $\begin{array}{l}\text { The Journal of } \\
\text { Nutrition, Health \& } \\
\text { Aging }\end{array}$ & $\begin{array}{l}\text { Avaliar a associação entre o excesso de peso } \\
\text { e a obesidade abdominal em idosos de } 80 \\
\text { anos ou mais }\end{array}$ \\
\hline $\begin{array}{l}\text { Downer, B., et al. } \\
\text { (2016). México (14) }\end{array}$ & $\begin{array}{c}\text { Journal of Aging and } \\
\text { Health }\end{array}$ & $\begin{array}{l}\text { Examinar a saúde dos mexicanos americanos } \\
\text { e mexicanos com idade } \geq 80 \text { anos }\end{array}$ \\
\hline $\begin{array}{l}\text { IP, E., et al. (2017). } \\
\text { Austrália (15) }\end{array}$ & $\begin{array}{l}\text { Internal Medicine } \\
\quad \text { Journal }\end{array}$ & $\begin{array}{l}\text { Descrever o uso de quimioterapia paliativa } \\
\text { de primeira linha em pacientes com } \geq 80 \\
\text { anos }\end{array}$ \\
\hline $\begin{array}{l}\text { Fakhry, N., et al. } \\
\text { (2013). França (16) }\end{array}$ & $\begin{array}{l}\text { International Journal } \\
\text { of Oral and } \\
\text { Maxillofacial Surgery }\end{array}$ & $\begin{array}{l}\text { Avaliar os resultados cirúrgicos de pacientes } \\
\text { com } \geq 80 \text { anos, operados de neoplasias } \\
\text { parotídeas }\end{array}$ \\
\hline $\begin{array}{l}\text { Garrouste-Orgeas, M., } \\
\text { et al. (2013). França } \\
\text { (17) }\end{array}$ & $\begin{array}{l}\text { Intensive Care } \\
\text { Medicine }\end{array}$ & $\begin{array}{l}\text { Avaliar as decisões médicas para pacientes } \\
\text { com } \geq 80 \text { anos nas Unidades de Terapia } \\
\text { Intensiva }\end{array}$ \\
\hline
\end{tabular}




\begin{tabular}{|c|c|c|}
\hline $\begin{array}{l}\text { Gon } \\
(201\end{array}$ & rão & $\begin{array}{l}\text { Analisar a presença de fatores de risco } \\
\text { cardiovascular em brasileiros com } \geq 80 \text { anos }\end{array}$ \\
\hline 017). & Diges & $\begin{array}{l}\text { Avaliar a segurança e a eficácia das sedações } \\
\text { durante a colangiopancreatografia retrógrada } \\
\text { endoscópica }\end{array}$ \\
\hline $\begin{array}{l}\text { Hayashi, Y., et al. } \\
\text { (2017). Japão (20) }\end{array}$ & Radia & $\begin{array}{l}\text { Observar os efeitos da quimioterapia e } \\
\text { radioterapia no Carcinoma de células } \\
\text { escamosas orais }\end{array}$ \\
\hline $\begin{array}{l}\text { Gul } \\
\text { Reir }\end{array}$ & $\begin{array}{l}\text { Population Health } \\
\text { Metrics }\end{array}$ & $\begin{array}{l}\text { Avaliar as transições demográfic } \\
\text { em uma população com } \geq 80 \text { an }\end{array}$ \\
\hline $\begin{array}{l}\text { Hegendörfer, E., et al. } \\
\text { (2017a). Bélgica (22) }\end{array}$ & exp & $\begin{array}{l}\text { Investigar o valor prognóstico do declínio do } \\
\text { volume expiratório forçado para resultados } \\
\text { adversos }\end{array}$ \\
\hline & ge & $\begin{array}{l}\text { vestigar correlatos da dispneia e sua } \\
\text { sociação }\end{array}$ \\
\hline al. (2014). & $\begin{array}{l}\text { Heart, Lung } \\
\quad \text { Circulatic }\end{array}$ & $\begin{array}{l}\text { Avaliar os desfechos clínicos dos idosos } \\
\text { submetidos à cirurgia de reposição valvar } \\
\text { aórtica }\end{array}$ \\
\hline $\begin{array}{l}\text { Laribi, K., et al. (2016). } \\
\text { França (25) }\end{array}$ & $\begin{array}{l}\text { Annals of } \\
\text { Hematology }\end{array}$ & $\begin{array}{l}\text { Avaliar a eficácia e segurança da } \\
\text { doxorubicina, em pacientes com } \geq 80 \text { anos } \\
\text { com linfoma }\end{array}$ \\
\hline $\begin{array}{l}\text { Levir } \\
(2016\end{array}$ & $\begin{array}{r}\text { Internal } N \\
\text { Jour }\end{array}$ & $\begin{array}{l}\text { Determinar comprometimento funcional/ } \\
\text { qualidade de vida em idade } \geq 80 \text { anos } \\
\text { admitidos na Unidade de Terapia Intensiva }\end{array}$ \\
\hline et al., & $\begin{array}{r}\text { Australasian } \\
\text { on Age }\end{array}$ & $\begin{array}{l}\text { Avaliar a predição da mortalidade por Infarto } \\
\text { em pacientes chineses de } \geq 80 \text { anos } \\
\text { hospitalizado }\end{array}$ \\
\hline $\begin{array}{l}\text { Mion } \\
\text { Franç }\end{array}$ & $\begin{array}{r}\text { Ge } \\
\text { Gerc } \\
\text { Inter }\end{array}$ & $\begin{array}{l}\text { Avaliar o tratamento com ativador de } \\
\text { plasminogênio para pacientes mais velhos } \\
\text { com AVC isquêmico }\end{array}$ \\
\hline 2016). & Plos & em \\
\hline $\begin{array}{l}\text { Pego, } \\
(2016)\end{array}$ & $\begin{array}{r}\text { Journal } \\
\text { and ereb } \\
\text { Dis }\end{array}$ & $\begin{array}{l}\text { Comparar as taxas de complicações em } \\
\text { pacientes com } \geq 80 \text { anos e com trombólise } \\
\text { intravenosa }\end{array}$ \\
\hline et al. & & $\begin{array}{l}\text { ncia de hipotensão } \\
\text { com } \geq 80 \text { anos }\end{array}$ \\
\hline $\begin{array}{l}\text { Ragot, S., et al. } \\
\text { França (32) }\end{array}$ & $\begin{array}{l}\text { Archives of } \\
\text { Cardiovascular } \\
\quad \text { Disease }\end{array}$ & $\begin{array}{l}\text { Avaliar o manejo da hipertensão não } \\
\text { controlada em pacientes franceses com } \geq 80 \\
\text { anos }\end{array}$ \\
\hline NIIa & $\begin{array}{c}\text { Digestive and Live } \\
\text { Disease }\end{array}$ & $\begin{array}{l}\text { Estudo com pacientes muito idosos para } \\
\text { tratar câncer colorretal }\end{array}$ \\
\hline
\end{tabular}




\begin{tabular}{|c|c|c|}
\hline $\begin{array}{l}\text { Rossi, F. E., et al. } \\
\text { (2016). Brasil (34) }\end{array}$ & Motriz & $\begin{array}{l}\text { Parâmetros } \\
\text { hemodinâmicos/antropométricos/metabólicos } \\
\text { em idosos } \geq 80 \text { anos }\end{array}$ \\
\hline $\begin{array}{l}\text { Santos, V. R., et al. } \\
\text { (2013). Brasil (35) }\end{array}$ & $\begin{array}{l}\text { Revista Brasileira de } \\
\text { Ortopedia }\end{array}$ & $\begin{array}{l}\text { Analisar a associação da massa óssea com a } \\
\text { capacidade funcional de idosos de } 80 \text { anos } \\
\text { ou mais }\end{array}$ \\
\hline $\begin{array}{l}\text { Santos, V. R., et al. } \\
\text { (2015). Brasil (36) }\end{array}$ & Revista de Nutrição & $\begin{array}{l}\text { Analisar sarcopenia/ r fatores } \\
\text { sociodemográficos/ doenças crônicas não } \\
\text { transmissíveis idosos }\end{array}$ \\
\hline $\begin{array}{l}\text { Santos, V. R., et al. } \\
\text { (2016). Brasil (37) }\end{array}$ & $\begin{array}{l}\text { Diabetes \& Metabolic } \\
\text { Syndrome }\end{array}$ & $\begin{array}{l}\text { Analisar o perfil lipídico de idosos com } \\
\text { idade } \geq 80 \text { anos de acordo com a composição } \\
\text { corporal }\end{array}$ \\
\hline $\begin{array}{l}\text { Tokushige, K., et al. } \\
\text { (2015). Japão (38) }\end{array}$ & Hepatology Research & $\begin{array}{l}\text { Esclarecer as características clínicas dos } \\
\text { pacientes com carcinoma hepatocelular }\end{array}$ \\
\hline $\begin{array}{l}\text { Varga, C., et al. (2014). } \\
\text { Canadá (39) }\end{array}$ & $\begin{array}{l}\text { Journal Leukemia e } \\
\text { Lymphoma }\end{array}$ & $\begin{array}{l}\text { Comparar aspectos clínicos/ patológicos de } \\
\text { portadores de linfoma de célula B com } \geq 80 \\
\text { anos }\end{array}$ \\
\hline $\begin{array}{l}\text { Wang, H., et al. (2016). } \\
\text { China (40) }\end{array}$ & $\begin{array}{l}\text { Kidney \& Blood } \\
\text { Pressure Researh }\end{array}$ & $\begin{array}{l}\text { Avaliar o impacto da doença renal crônica } \\
\text { em hipertensos não tratados com } \geq 80 \text { anos } \\
\text { na China }\end{array}$ \\
\hline $\begin{array}{l}\text { Warwick, J. et al. } \\
\text { (2015). Reino Unido } \\
(41)\end{array}$ & BioMed Central & $\begin{array}{l}\text { Investigar se existe uma interação entre o } \\
\text { tratamento para hipertensão e fragilidade em } \\
\text { idosos }\end{array}$ \\
\hline $\begin{array}{l}\text { Wauters, M. et al. } \\
\text { (2017). Bélgica (42) }\end{array}$ & $\begin{array}{l}\text { Basic \& Clinical } \\
\text { Pharmacology \& } \\
\text { Toxicology }\end{array}$ & $\begin{array}{l}\text { Avaliar a prevalência e a intensidade da } \\
\text { exposição anticolinérgica de pacientes muito } \\
\text { idosos }\end{array}$ \\
\hline $\begin{array}{l}\text { Yoshida, M. et al. } \\
\text { (2017). Japão (43) }\end{array}$ & Surgical Endoscopy & $\begin{array}{l}\text { Avaliar a segurança e a eficácia da } \\
\text { gastrectomia distal assistida por laparoscopia } \\
\text { em pacientes idosos com } \geq 80 \text { anos }\end{array}$ \\
\hline $\begin{array}{l}\text { Yu, W. C., et al. (2017). } \\
\text { Taiwan (44) }\end{array}$ & Plos One & $\begin{array}{l}\text { Avaliar } \quad \text { inter-relação } \\
\text { deficiência } \\
\text { cognitivo na mortalidade }\end{array}$ \\
\hline
\end{tabular}

\section{Análise dos trabalhos encontrados}

Em relação aos autores, houve certa homogeneidade nas áreas de conhecimento sendo $(58,3 \%)$ procedente da área médica. Destes, $13,8 \%$ é correspondente a pesquisas publicadas na especialidade de Gastroenterologia. Portanto, ainda encontramos muitos estudos médico-centrados voltados para o controle de doenças e medicalização. Apenas dois artigos eram oriundos de Centros de Geriatria e Gerontologia $(5,4 \%)$. 
Em síntese, foram encontrados cinco periódicos que se referem ao envelhecimento, sendo eles: The Journal of Nutrition, Health \& Aging, Journal of Aging and Health; Aging Clinical and Experimental Research; Internal Medicine Journal e Australasian Journal and Ageing. Dentre todos os periódicos encontrados, apenas quatro são nacionais: Revista Medicina de Ribeirão Preto, Revista Motriz, Revista Brasileira de Ortopedia e a Revista de Nutrição.

Em relação aos objetivos do estudo, dos 36 artigos analisados, 27\% sinalizaram para intervenção medicamentosa, tanto na área experimental quanto na análise comparativa de estudos já realizados na área da saúde. Padrões antropométricos e hemodinâmicos, problemas como hipertensão, diabetes, entre outras afecções presentes nos idosos, foram abordados em $22 \%$ dos estudos, enquanto $16 \%$ estava direcionado a desvelar a capacidade funcional e sua relação com a qualidade de vida dos idosos longevos. Os demais estudos consideraram, em seus objetivos, intervenções cirúrgicas óssea, gastrointestinal e respiratória, entre outros.

Entre os objetivos contemplados nos artigos estudados, destacam-se as doenças, sendo as mais estudadas as neoplasias, em oito artigos (Ip, 2017; Fakhry, et al., 2013; Hayashi, et al., 2017; Laribi, et al., 2016; Park, et al., 2016; Rinaldi, et al., 2017; Tokushige, Hashimoto, Horie, Taniai, \& Higuchi, 2014; e Varga, et al., 2014). Esses dados justificam, em parte, os achados no que diz respeito aos estudos sobre intervenção medicamentosa, tendo em vista que, em sua maioria, tratavam-se de quimioterápicos (Ip, 2017; Hayashi, et al., 2017; Laribi, et al., 2016).

Nove artigos abordavam problemas cardíacos (David, Mello, Bruscato, \& Moriguchi, 2016; Gomes, et al., 2016; Ho, 2014; Luo, et al., 2014; Pérez-Orcero, et al., 2016; Ragot, Sosner, Dievart, \& Herpin, 2014; Rossi, et al., 2016; Wang, et al., 2016; Warwick, et al., 2015), incluindo dados hemodinâmicos e antropométricos como procedimentos a serem utilizados no controle de algumas patologias como hipertensão e outras. Reitera-se a ênfase dos pesquisadores sobre investigações relacionadas aos agravos mais frequentes na saúde do idoso longevo e respectivas terapêuticas.

Quanto ao delineamento das publicações, verifica-se o predomínio de estudos transversais (41,6\%); seguidos por estudos de coorte $(25,0 \%)$; estudos caso-controle $(16,6 \%)$; e ensaios clínicos randomizados (16,6\%). Ressalta-se que os estudos epidemiológicos transversais são os mais usados, pois permitem avaliar associações 
entre exposições e desfechos, embora não possibilitem uma avaliação de causa e efeito (Medronho, Bloch, Raggio, Ronir, \& Loureiro, 2009).

A despeito de a maioria dos estudos ser do tipo transversal, existe um aumento da produção de pesquisas longitudinais, com amostras maiores, o que possibilita conclusões mais adequadas sobre as mudanças intra e interindividuais que ocorrem na velhice e no processo de envelhecimento (Guariento, Neri, Fattori, \& Pereira, 2013). Uma das possíveis soluções para a expansão das pesquisas com idosos longevos no Brasil, para além de estudos transversais, seria a formação de grupos transnacionais e a institucionalização da cooperação internacional, que auxiliaria na redução de custos e aumentaria o apoio técnico especializado essencial na elaboração de estudos de coorte (Guariento, Neri, Fattori, \& Pereira, 2013).

Entre os estudos transversais desta revisão, um estudo avaliou que a massa óssea do fêmur para idosos longevos do sexo masculino está associada à capacidade funcional (Santos, et al., 2013) e, em outro estudo, observamos que a obesidade está associada à alta prevalência de fatores de risco cardiovascular (Gomes, et al., 2016).

Ainda sobre o tipo de delineamento dos estudos, nota-se que, apesar das bases de dados utilizadas na pesquisa apresentarem estudos do tipo qualitativo, na presente revisão não foi encontrado nenhum artigo com este tipo de formato. Devemos nos atentar, visto que os estudos qualitativos possibilitam o levantamento de dados sobre as motivações de um grupo em compreender e interpretar determinados comportamentos, hábitos, atitudes, a opinião e as expectativas dos indivíduos de uma população, o que no caso dos idosos longevos seria uma grande contribuição para o entendimento dos aspectos psicológicos e sociais vivenciados nesta fase da vida (Lakatos, \& Marconi 2011).

Em relação ao número de participantes (amostra) dos estudos considerados, 13,8\% continha menos de 100 idosos, 36,1\% continha entre 101 a 250 participantes, $22,2 \%$ entre 251 a 500; e, em 27,7\% dos estudos, acima de 500 indivíduos. Portanto, a maioria dos estudos contou com uma amostra de 101 a 250 participantes (13 artigos). Na revisão realizada, foram encontrados seis estudos nacionais (David, Mello, Bruscato, \& Moriguchi, 2016; Gomes, et al., 2016; Rossi, et al., 2016; Santos, 2013; 2015; 2016), todos com uma amostra inferior a 500 participantes. Os artigos com mais representatividade de idosos são os oriundos de países europeus, aqueles realizados no 
Japão, ou estudos multicêntricos internacionais. Cabe ressaltar a importância do tamanho da amostra, podendo limitar a obtenção de informações se não for representativa.

Apenas $20 \%$ dos estudos incluídos nesta revisão não considerou exclusivamente os idosos com idade igual ou superior a 80 anos. Estudos direcionados apenas a este subgrupo são muito importantes para que haja uma melhor tomada de decisão quanto ao melhor direcionamento de cuidados para estes indivíduos (Guariento, Neri, Fattori, \& Pereira, 2013).

Em relação à distribuição amostral de acordo com o sexo, as mulheres representaram a maioria (61\%), o que denota a feminização da velhice, fenômeno que ocorre devido ao crescimento da população feminina em taxas mais elevadas, associado à menor mortalidade deste subgrupo nas faixas etárias anteriores (Guariento, Neri, Fattori, \& Pereira, 2013).

Quanto ao método de coleta de dados utilizado nos estudos pesquisados, verificamos uma grande diversidade de instrumentos (31 tipos diferentes). Os instrumentos mais encontrados nesta revisão relacionaram-se à avaliação funcional, em nove artigos (Bonicoli, et al., 2016; Downer, Chen, Wong, \& Markides, 2016; Hegendörfer, et al., 2017b; Levinson, et al., 2016; Pego, Nunes, Ferreira, Sousa, \& Amaral-Silva,, 2016; Wang, et al., 2016; Warwick, et al., 2015; Yoshida, et al., 2017; Yu, et al., 2017) e instrumentos preditores de risco, em sete artigos (Hegendörfer, et al., 2017b; Levinson, et al., 2016; Luo, et al., 2014; Mione, et al., 2016; Pego, Nunes, Ferreira, Sousa, \& Amaral-Silva, 2016; Rinaldi, et al., 2017; Yoshida, et al., 2017). Para Paixão (2005), a definição da avaliação do estado funcional seria "o nível com o qual a pessoa desempenha funções e atividades da vida diária". O objetivo de identificar, mais precisamente, os idosos em risco permite estabelecer uma estratificação dessa população. Assim, a chance estimada de adoecimento ou óbito possibilita decisões que organizem as intervenções assistenciais e preventivas, ou ainda que admitam adequada alocação de recursos humanos ou financeiros (Estrella, Oliveira, Sant'Anna, \& Caldas, 2009).

Para aprofundar a análise em relação às escalas funcionais, no presente estudo, foram agrupadas em escalas de avaliação cognitiva (Mini-Exame do Estado Mental, escala Short Portable Mental Status Questionnaire (SPMSQ) e escala de Glasgow); 
escalas de avaliação de atividades da vida diária, atividades básicas de vida diária e atividades instrumentais da vida diária (Índice de Barthel, Índice de Katz, Escala de Lawnton e Brody, respectivamente); e escala física Physical Self-Maintenance Scale (PSMS). Dos dez artigos que utilizaram as escalas funcionais, as mais usadas foram o Mini-Exame do Estado Mental na avaliação cognitiva de três artigos (Bonicoli, et al., 2016; Wang, et al., 2016; Yu, et al., 2017).

Também foi possível observar que a coleta de dados sobre os aspectos clínicos dos idosos são predominantes nos artigos selecionados neste estudo. Seis artigos avaliaram parâmetros metabólicos dos indivíduos (resultados de exames de colesterol, glicose, creatinina etc.) (Bourguignon, et al., 2016; Bruyère, et al., 2014; Gomes, et al., 2016; Santos, 2016; Varga, et al., 2014; Wang, et al., 2016); seis consideraram os parâmetros antropométricos dos idosos (Indice de Massa Corporal, peso, altura etc.) (Bourguignon, et al., 2016; David, Mello, Bruscato, \& Moriguchi, 2016; Gomes, et al., 2016; Pérez-Orcero, et al., 2016; Santos, et al., 2016; Wang, et al., 2016) e quatro artigos, os parâmetros de composição corporal, obtidos por densitometria por emissão de raios X de dupla energia (DXA) (Gomes, et al., 2016; Santos, et al., 2013; 2015; 2016), por meio de estudos experimentais.

\section{Considerações Finais}

Os resultados obtidos nesta revisão possibilitaram identificar o que vem sendo abordado nas publicações científicas sobre os idosos com $\geq 80$ anos. Destaca-se a importância de identificar as lacunas de conhecimento, pois, com o envelhecimento populacional dos idosos mais idosos, os indivíduos são acometidos por várias doenças crônicas e expostos frequentemente ao uso exacerbado de medicações, havendo a necessidade de investigação desses temas neste subgrupo específico.

\section{Referências}

Baltes, P. B., \& Smith, J. (2006). Novas fronteiras para o futuro do envelhecimento: a velhice bem-sucedida do idoso jovem aos dilemas da quarta idade. A terceira idade, 17(36), 7-31. Recuperado em 01 dezembro, 2017, de: https://www.sescsp.org.br/files/edicao_revista/4ed8a079-074e-4baf-8f72-6770562f0853.pdf. 
Bode, B., Stenlöf, K., Harris, S., Sullivan, D., Fung, A., Usiskin, K., et al. (2015) Longterm efficacy and safety of canagliflozin over 104 weeks in patients aged 55-80 years with type 2 diabetes. Diabetes, Obesidade e Metabolismo, 17(3), 294-303. Recuperado em 01 dezembro, 2017, de: doi: 10.1111/dom.12428.

Bonicoli, E., Niccolai, F., Pasqualetti, G., Bini, G., Monzani, F., \& Lisanti, M. (2016). The difference in activity of daily living (ADL) and mortality in patients aged over 80 years with femoral neck fracture treated with hemiarthroplasty or osteosynthesis at 2 years of follow-up. Injury. Elsevier, 47, S112-5. Recuperado em 16 de outubro, 2018, de: http://dx.doi.org/10.1016/j.injury.2016.07.036.

Bourguignon, L., Cazaubon, Y., Debeurme, G., Loue, C., Ducher, M., \& Goutelle, S. (2016). Pharmacokinetics of vancomycin in elderly patients aged over 80 years. Antimicrobial Agents and Chemotherapy, 60(8), 4563-4567. Recuperado em 16 de outubro, 2018, de: 10.1128/AAC.00303-16.

Bruyère, O., Slomian, J., Beaudart, C., Buckinx, F., Cavalier, E., Gillain, S., et al., (2014). Prevalence of vitamin D inadequacy in European women aged over 80 years. Archives of Gerontology and Geriatrics, 59(1), 78-82. Recuperado em 01 dezembro, 2017, de: doi: 10.1016/j.archger.2014.03.010.

David, C. N., Mello, R. B., Bruscato, N. M., \& Moriguchi, E. H. (2016). Overweight and Abdominal Obesity Association with All-Cause and Cardiovascular Mortality in the Elderly Aged 80 and Over: a Cohort Study. The Journal of Nutrition Health and Aging, 21, 1-7. Recuperado em 16 de outubro, 2018, de: DOI: 10.1007/s12603-016-0812-0.

Downer, B., Chen, N. W., Wong, R., \& Markides, K. (2016). Self-Reported Health and Functional Characteristics of Mexican and Mexican American Adults Aged 80 and Over. Journal of Aging and Health, 28(7), 1-15. Recuperado em 16 de outubro, 2018, de: DOI: $10.1177 / 0898264316656508$.

Estrella, K., Oliveira, C. E. F., Sant'Anna, A. A., \& Caldas, C. P. (2009). Detecção do risco para internação hospitalar em população idosa: um estudo a partir da porta de entrada no sistema de saúde suplementar. Cadernos de Saúde Pública, 25(3), 507-512. Recuperado em $16 \quad$ outubro, 2018, de: http://www.scielo.br/scielo.php?script=sci_arttext\&pid=S0102-311X2009000300005\& $\operatorname{lng}=\mathrm{pt} \& \operatorname{lng}=\mathrm{pt}$.

Fakhry, N., Aldosari, B., Michel, J., Giorgi, R., Collet, C., Santini, L., et al. (2013). Clinical and oncological outcomes after surgical excision of parotid gland tumours in patients aged over 80 years. International Journal of Oral and Maxillofacial Surgery, 42(11), 1385-1390. Recuperado em 16 outubro, 2018, de: http://dx.doi.org/10.1016/j.ijom.2013.06.010.

Garrouste-Orgeas, M., Tabah, A., Vesin, A., Philippart, F., Kpodji, A., Bruel, C., et al. (2013). The ETHICA study (part II): Simulation study of determinants and variability of ICU physician decisions in patients aged 80 or over. Intensive Care Medicine, 39(9), 1574-1583. Recuperado em 01 dezembro, 2017, de: DOI: 10.1007/s00134-013-2977-x.

Gil, A. C. (2002). Como Elaborar Projetos de Pesquisa. São Paulo, SP: Atlas. 
Gomes, I. C., Santos, V. R., Christofaro, D. G., Fernandes, R. A., Bueno, D. R., \& Freitas Júnior, I. F. (2016). Cardiovascular risk factors and body fat distribution in brazilians aged 80 years or over. Ribeirão Preto Online, 49(1), 17-25. Recuperado em 16 outubro, 2018, de: http://revista.fmrp.usp.br/2016/vol49n1/AO3-Cardiovascular-riskfactors-and-body-fat-distribution-in-the-oldest-elderly.pdf.

Guariento, M. E., Neri, A. L., Fattori, A., \& Pereira, A. P. (2013). Pesquisa em Gerontologia. In: Freitas, E. V., \& Py, L. (Eds.). Tratado de geriatria e gerontologia. ( $3^{\mathrm{a}}$ ed.). Rio de Janeiro, RJ: Guanabara Koogan.

Han, S. J., Lee, T. H., Park, S. H., Cho, Y. S., Lee, Y. N., Jung, Y., et al. (2017). Efficacy of midazolam- versus propofol-based sedations by non-anesthesiologists during therapeutic endoscopic retrograde cholangiopancreatography in patients aged over 80 years. Endoscopy, 29(3), 369-376. Recuperado em 16 outubro, 2018, de: DOI: $10.1111 /$ den.12841.

Hayashi, Y., Mitsudo, K., Sakuma, K., Iida, M., Iwai, T., Nakashima, H., et al. (2017). Clinical outcomes of retrograde intra-arterial chemotherapy concurrent with radiotherapy for elderly oral squamous cell carcinoma patients aged over 80 years old. Radiation Oncology, 12(1), 112. Recuperado em 16 outubro, 2018, de: http://rojournal.biomedcentral.com/articles/10.1186/s13014-017-0847-3.

Hazra, N. C., \& Gulliford, M. (2017). Evolution of the "fourth stage" of epidemiologic transition in people aged 80 years and over: population-based cohort study using electronic health records. Population Health Metrics, 15(1), 18. Recuperado em 16 outubro, 2018, de: http://pophealthmetrics.biomedcentral.com/articles/10.1186/s12963017-0136-2.

Hegendörfer, E., Vaes, B., Matheï C., Van., Pottelbergh, G., \& Degryse, J. M. (2017a). Correlates of dyspnea and its association with adverse outcomes in a cohort of adults aged 80 and over. Age and Ageing, 46(6), 994-1000. Recuperado em 16 outubro, 2018, de: http://www.ncbi.nlm.nih.gov/pubmed/28633384.

Hegendörfer, E., Vaes, B., Matheï, C., Van., Pottelbergh, G., \& Degryse, J. M. (2017b). Prognostic value of short-term decline of forced expiratory volume in $1 \mathrm{~s}$ over height cubed $(\mathrm{FEV} 1 / \mathrm{Ht} 3)$ in a cohort of adults aged 80 and over. Aging Clinical and Experimental Research, 1-10. Recuperado em 16 outubro, 2018, de: https://europepmc.org/abstract/med/28653254.

Ho, E., Mathur, M.N., Brady, P. W., Marshman, D., Brereton, R. J., Ross, D. E., et al. (2014). Surgical aortic valve replacement in very elderly patients aged 80 years and over: Evaluation of early clinical outcomes. Heart, Lung and Circulation, 23(3), 242248. Recuperado em 16 outubro, 2018, de: http://dx.doi.org/10.1016/j.hlc.2013.08.001.

IBGE. (2015). Mudança Demográfica no Brasil no início do século XXI: Subsídios para as projeções da população. Rio de Janeiro, RJ: IBGE.

Ip, E., Pokorny, A. M. J., Della-Fiorentina S., Beale P., Bray V., Kiely B. E., et al. (2017). Use of palliative chemotherapy in patients aged 80 years and over with incurable cancer: experience at three Sydney cancer centres. Internal Medicine Journal, 47(1), 75-81. Recuperado em 16 outubro, 2018, de: DOI: 10.1111/imj.13296. 
Kumon, M. T., Silva, V. P., Silva, A. I., \& Gomes, L. (2009). Centenários no mundo: uma visão panorâmica. São Paulo, SP: PUC-SP: Revista Kairós-Gerontologia, 12(1). ISSNprint 1516-2567. ISSNe 2176-901X. Recuperado em 16 outubro, 2017, de: https://revistas.pucsp.br/index.php/kairos/article/view/2788.

Lakatos, E. M., \& Marconi, M. A. (2011). Metodologia científica. (6ª ed.). São Paulo, SP: Atlas.

Laribi, K., Denizon, N., Bolle, D., Truong, C., Besançon, A., Sandrini, J., et al. (2016). $\mathrm{R}-\mathrm{CVP}$ regimen is active in frail elderly patients aged 80 or over with diffuse large B cell lymphoma. Annals of Hematology, 95(10), 1705-1714. Recuperado em 16 outubro, 2018, de: http://dx.doi.org/10.1007/s00277-016-2768-x.

Levinson, M., Mills, A., Oldroyd, J., Gellie, A., Barrett, J., Staples, M., et al. (2016). The impact of intensive care in a private hospital on patients aged 80 and over: Healthrelated quality of life, functional status and burden versus benefit. International Journal of Medicine, 46(6), 694-702. Recuperado em 01 dezembro, 2017, de: doi: 10.1111/imj.13079.

Lima, W.R. (2014). Estilo de vida e sua associação com a longevidade de idosos muito velhos de Aracaju, SE. Biblioteca Digital de Teses e Dissertações da Universidade de São Paulo. Recuperado em 16 de outubro, 2018, de: http://www.teses.usp.br/teses/disponiveis/83/83131/tde-26062014-121118/.

Luo, J., Yang, M., Han, L., Chen, L., Chen, X., Gao, K., et al. (2014). Validity of the Global Registry of Acute Coronary Events risk score in prediction of acute myocardial infarction mortality in hospitalised Chinese patients aged 80 and over. Australasian Journal on Ageing, 33(4), E1-5. Recuperado em 16 outubro, 2018, de: http://doi.wiley.com/10.1111/ajag.12044.

Medronho, R. A., Bloch, K. V., Raggio, L., Ronir, W., \& Loureiro, G. (2009). Epidemiologia. Rio de Janeiro, RJ: Editora Atheneu.

Mione, G., Ducrocq, X., Thilly, N., Lacour, J.C., Vespignani, H., \& Richard, S. (2016). Outcome of intravenous recombinant tissue plasminogen activator for acute ischemic stroke in patients aged over 80 years. Geriatriacs \& Gerontology International, 16(7), 843-849. Recuperado em 01 dezembro, 2017, de: https://doi.org/10.1111/ggi.12565.

Souza, M. T., Silva, M. D., \& Carvalho, R. (2010). Revisão integrativa: o que é e como fazer. Einstein, 8(1), 102-106. Recuperado em 24 de julho, 2019, de: http://www.scielo.br/pdf/eins/v8n1/pt_1679-4508-eins-8-1-0102.pdf.

Organização Mundial da Saúde (OMS). (2004). Classificação Internacional de funcionalidade, Incapacidade e Saúde (CIF), 8. Lisboa, Portugal.

Organização Mundial da Saúde (OMS). (2015). Envelhecimento ativo: uma política de saúde. Brasília, DF: Organização Pan Americana de Saúde (62 p.).

Paixão Jr, C.M., Reichenheim, M.E. (2005). Uma revisão sobre instrumentos de avaliação do estado funcional do idoso. Cadernos de Saúde Pública, 21(1), 7-19. Recuperado em $16 \quad$ outubro, 2018, de: http://www.scielo.br/scielo.php?script=sci_arttext\&pid=S0102-

$311 \times 2005000100002 \& \operatorname{lng}=$ pt\&tlng=pt. 
Park, H. J., Ahn, J. Y, Jung, H. Y., Lee, J. H, Jung, K. W., Kim, D. H., et al. (2016). Clinical Characteristics and Outcomes of Gastric Cancer Patients Aged over 80 Years: A Retrospective Case-Control Study. PLOS One, 11(12). Recuperado em 16 outubro, 2018, de: https://www.ncbi.nlm.nih.gov/pubmed/27942044.

Pego, P. M., Nunes, A. P., Ferreira, P., Sousa, C., \& Amaral-Silva, A. (2016). Thrombolysis in patients aged over 80 years is equally effective and safe. Elsevier, 25(6), 1532-1538. Recuperado em 16 outubro, 2018, de: http://dx.doi.org/10.1016/j.jstrokecerebrovasdis.2016.03.007.

Pérez, O. A., Vinyoles, B. E., Pujol, R. E., Figuera, V., Wichmann, M., Baena, D. J. M., Manjón, V. R., et al. (2016). Prevalence of orthostatic hypotension in noninstitutionalised elderly aged 80 and over. A diagnostic study using an oscillometric device. Hipertension y Riesgo Vascular, 33(3), 93-102. Recuperado em 16 outubro, 2018, de: http://linkinghub.elsevier.com/retrieve/pii/S188918371600026X.

Ragot, S., Sosner, P., Dievart, F., \& Herpin, D. (2014). Prevalence and management of uncontrolled hypertension in French patients aged over 80 years. Archives of Cardiovascular Disease, 107(4), 236-244. Recuperado em 16 outubro, 2018, de: https://www.em-consulte.com/en/article/898186.

Rinaldi, L., Ouaissi, M., Barabino, G., Loundou, A., Clavel, L., Sielezneff, I., et al. (2017). Laparoscopy could be the best approach to treat colorectal cancer in selected patients aged over 80 years: Outcomes from a multicenter study. Digestive and Liver Disease, 49(1), 84-90. Recuperado em 16 outubro, 2018, de: http://dx.doi.org/10.1016/j.dld.2016.06.039

Rossi, F. E., Ricci-Vitor, A. L., Gomes, I. C., Santos, V. R., Sabino, J. P. J., Branco, L. G. S., et al. (2016). Relationship between resting heart rate and anthropometric, metabolic and hemodynamic parameters in the elderly aged 80 years and over. Motriz: Revista de Educação Física, 22(1), 18. Recuperado em 01 dezembro, 2017, de: http://dx.doi.org/10.1590/S1980-65742016000100003.

Santos, V. R., Christofaro, D. G. D., Gomes, I. C., Codogno, J. S., Santos, L. L., \& Freitas, J. I. F. (2013). Associação entre massa óssea e capacidade funcional de idosos com 80 anos ou mais. Revista Brasileira de Ortopedia, 48(6), 512-518. Recuperado em 01 dezembro, 2017, de: http://dx.doi.org/10.1016/j.rboe.2013.12.012.

Santos, V. R., Christofaro, D. G. D., Gomes, I. C., Agostinete, R. R., Freitas, J. I. F., \& Gobbo, L. A. (2015). Factors associated with sarcopenia in subjects aged 80 years and over. Revista de Nutrição, 28(3), 319-326. Recuperado em 01 dezembro, 2017, de: http://dx.doi.org/10.1590/1415-52732015000300008.

Santos, V. R., Christofaro, D. G. D., Gomes, I. C., Viezel, J., Freitas, J. I. F., \& Gobbo, L. A. (2016). Analysis of relationship of high fat mass and low muscle mass with lipid profile in Brazilians aged 80 years or over. Diabetes \& Metabolic Syndrome: Clinical Researchs and Reviews, 10(5). Recuperado em 16 outubro, 2018, de: http://linkinghub.elsevier.com/retrieve/pii/S1871402116302557. 
Tokushige, K., Hashimoto, E., Horie, Y., Taniai, M., \& Higuchi, S. (2014). Hepatocellular carcinoma based on cryptogenic liver disease: The most common nonviral hepatocellular carcinoma in patients aged over 80 years. Hepatology Research, 1(7). Recuperado em 16 outubro, 2018, de: http://www.ncbi.nlm.nih.gov/pubmed/24923363.

United Nations (UN). (2013). World Population Prospects: The 2012 Revision, v. I: Comprehensive Tables ST/ESA/SER.A/336. New York, USA.

Varga, C., Holcroft, C., Kezouh, A., Bucatel, S., Johnson, N., Petrogiannis, H. T., et al. (2014). Comparison of outcomes among patients aged 80 and over and younger patients with diffuse large B-cell lymphoma: a population based study. Leukemia \& Lymphoma, 55(3), 533-537. Recuperado em 16 outubro, 2018, de: http://www.tandfonline.com/doi/full/10.3109/10428194.2013.790968.

Wang, H., Liu, T., Cai, Y., Jiang, H., Liu, H., \& Lin, C. (2016). Kidney Function and Cognitive Impairment in People Aged 80 Years and Over with Untreated Hypertension: A Cross-Sectional Survey. Kidney and Blood Pressure Research, 41(1), 70-77. Recuperado em 16 outubro, 2018, de: https://www.karger.com/Article/FullText/368550.

Warwick, J., Falaschetti, E., Rockwood, K., Mitnitski, A., Thijs, L., Beckett, N., et al. (2015). No evidence that frailty modifies the positive impact of antihypertensive treatment in very elderly people: an investigation of the impact of frailty upon treatment effect in the Hypertension in the Very Elderly Trial (HYVET) study, a double-blind, placeb. BMC Medicine, 13(1), 78. Recuperado em 16 outubro, 2018, de: http://bmcmedicine.biomedcentral.com/articles/10.1186/s12916-015-0328-1.

Wauters, M., Klamer, T., Elseviers, M., Vaes, B., Dalleur, O., Degryse, J., et al. (2017). Anticholinergic Exposure in a Cohort of Adults Aged 80 years and Over. Basic and Clinical Pharmacology and Toxicology, 120(6), 591-600. Recuperado em 16 de outubro, 2018: https://www.ncbi.nlm.nih.gov/pubmed/27995743.

Yoshida, M., Koga, S., Ishimaru, K., Yamamoto, Y., Matsuno, Y., Akita, S., et al. (2017). Laparoscopy-assisted distal gastrectomy is feasible also for elderly patients aged 80 years and over: effectiveness and long-term prognosis. Surgical Endoscopy, 31(11), 4431-4437. Recuperado em 16 outubro, 2018, de: doi: 10.1007/s00464-017-5493-1.

Yu, W. C., Chou, M. Y., Peng, L. N., Lin, Y.T., Liang, C. K., \& Chen, L. K. (2018). Synergistic effects of cognitive impairment on physical disability in all-cause mortality among men aged 80 years and over: Results from longitudinal older veterans study. PLOS One, 12(7). Recuperado em 16 outubro, 2018, de: https://www.ncbi.nlm.nih.gov/pubmed/28746360.

Recebido em 17/10/2018

Aceito em 30/01/2019 
Priscila de Paula Marques - Enfermeira. Doutoranda do Programa de Pós-Graduação em Gerontologia, Faculdade de Ciências Médicas, FCM, Universidade Estadual de Campinas, UNICAMP. Mestre em Saúde Coletiva, Política e Gestão em Saúde, Unicamp. Apoiadora Institucional na Prefeitura Municipal de Campinas.

URL: https://orcid.org/0000-0002-7954-4056

E-mail: enf.primarques@gmail.com

Carolina Neves Freiria - Nutricionista. Doutoranda do Programa de Pós-Graduação em Gerontologia, Faculdade de Ciências Médicas, FCM, Universidade Estadual de Campinas, UNICAMP. Mestra em Gerontologia.

E-mail: carol_freiria@hotmail.com

Caroline Nunes Santiago - Enfermeira. Mestranda do Programa de Pós-Graduação em Gerontologia, Faculdade de Ciências Médicas, FCM, Universidade Estadual de Campinas, UNICAMP. Especialista em Envelhecimento, Universidade Federal de São Paulo. Atualmente é enfermeira do Hospital Municipal Doutor Mario Gatti.

E-mail: caroline.nsantiago@ hotmail.com

Matilde Maria de Magalhães Arena Côrrea - Psicóloga. Mestra em Educação. Doutoranda do Programa de Pós-Graduação em Gerontologia, Faculdade de Ciências Médicas, FCM, Universidade Estadual de Campinas, UNICAMP. Professora efetiva da Rede Municipal de Educação da cidade de Campinas, atuando na CEI Carrossel. E-mail: mmatildinha@gmail.com 
Sandra Aparecida Bratifische - Graduação em Educação Física. Mestrado em Pedagogia do Movimento. Doutoranda do Programa de Pós-Graduação em Gerontologia, Faculdade de Ciências Médicas, FCM, Universidade Estadual de Campinas, UNICAMP. Professora do curso de Educação Física da Faculdade Santa Bárbara d'Oeste (Grupo Universidade Brasil). Professora das disciplinas de: Metodologia de Pesquisa, Atividade Física Gerontológica, Trabalho de Conclusão de Curso, Estágio e Dança.

E-mail sanbrati@yahoo.com.br

Priscila Maria Stolses Bergamo Francisco - Bacharel em Estatística. Mestre, Doutora e Pós-Doutora em Saúde Coletiva na área de Epidemiologia, pela Faculdade de Ciências Médicas da Unicamp. Professora Doutora, vinculada à área de Epidemiologia do Departamento de Saúde Coletiva. Pesquisadora plena credenciada aos Programas de Pós-Graduação em Saúde Coletiva, Pós-Graduação em Gerontologia e Mestrado Profissional em Saúde Coletiva, Política e Gestão em Saúde da Faculdade de Ciências Médicas da UNICAMP.

E-mail: primaria@fcm.unicamp.br

Maria José D'Elboux - Enfermeira; Mestre e Doutora em Enfermagem; LivreDocência pela Universidade Estadual de Campinas. Atualmente é professor colaborador da Universidade Estadual de Campinas, Programa de Pós-Graduação em Gerontologia, da Faculdade de Ciências Médicas, FCM, UNICAMP.

E-mail: mariadio@uol.com.br 